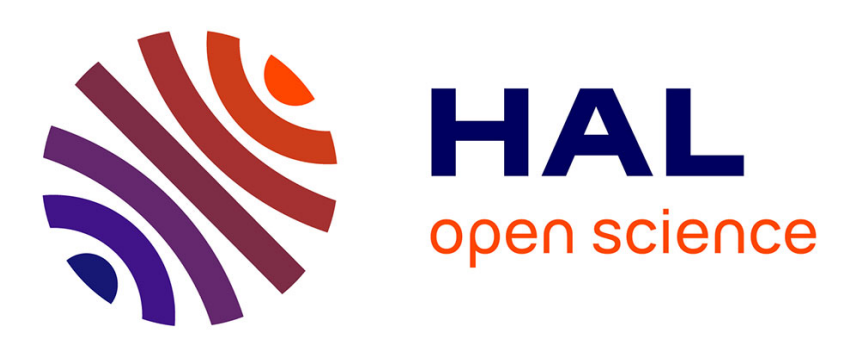

\title{
A pressure-correction scheme for convection-dominated incompressible flows with discontinuous velocity and continuous pressure
}

Lorenzo Botti, Daniele Antonio Di Pietro

\section{- To cite this version:}

Lorenzo Botti, Daniele Antonio Di Pietro. A pressure-correction scheme for convection-dominated incompressible flows with discontinuous velocity and continuous pressure. Journal of Computational Physics, 2010, 230 (3), pp.572-585. 10.1016/j.jcp.2010.10.004 . hal-00458293v2

\section{HAL Id: hal-00458293 \\ https://hal.science/hal-00458293v2}

Submitted on 1 Sep 2010

HAL is a multi-disciplinary open access archive for the deposit and dissemination of scientific research documents, whether they are published or not. The documents may come from teaching and research institutions in France or abroad, or from public or private research centers.
L'archive ouverte pluridisciplinaire HAL, est destinée au dépôt et à la diffusion de documents scientifiques de niveau recherche, publiés ou non, émanant des établissements d'enseignement et de recherche français ou étrangers, des laboratoires publics ou privés. 


\title{
A pressure-correction scheme for convection-dominated incompressible flows with discontinuous velocity and continuous pressure
}

\author{
Lorenzo Botti ${ }^{\mathrm{a}, \mathrm{b}, *}$, Daniele A. Di Pietro ${ }^{\mathrm{c}}$ \\ ${ }^{a}$ Biomedical Engineering Department, Mario Negri Institute for Pharmacological \\ Research, Bergamo, Italy \\ ${ }^{b}$ Industrial Engineering Department, University of Bergamo, Italy \\ ${ }^{c}$ Insitut Français du Pétrole, 1834 avenue Bois Préau, 92852 Rueil-Malmaison, France
}

\begin{abstract}
In this work we present a pressure-correction scheme for the incompressible Navier-Stokes equations combining a discontinuous Galerkin approximation for the velocity and a standard continuous Galerkin approximation for the pressure. The main interest of pressure-correction algorithms is the reduced computational cost compared to monolithic strategies. In this work we show how a proper discretization of the decoupled momentum equation can render this method suitable to simulate high Reynolds regimes. The proposed spatial velocity-pressure approximation is LBB stable for equal polynomial orders and it allows adaptive $p$-refinement for velocity and global $p$-refinement for pressure. The method is validated against a large set of classical two- and three-dimensional test cases covering a wide range of Reynolds numbers, in which it proves effective both in terms of accuracy and computational cost.
\end{abstract}

Keywords:

Incompressible Navier-Stokes equations, Discontinuous Galerkin, Pressure-Correction

\section{Introduction}

Discontinuous Galerkin ( $\mathrm{dG}$ ) methods constitute an effective means to obtain accurate discretizations of complex problems on general meshes. In this work, we deal with convection-dominated incompressible flows, which constitute a challenging class of problems both in terms of numerical stability and

\footnotetext{
*Send correspondence to Lorenzo Botti, viale Marconi 5 - 24044 Dalmine (BG) Fax:+39 035 2052077, Tel:+39 035 2052084, email Iorenzo.botti@unibg.it
} 
computational cost. In this context, dG methods offer many advantages: stabilized equal-order discretizations can be devised, the extension to arbitrary unstructured and nonconforming grids is straightforward, and the resulting discretization displays an increased stability in the high Reynolds regimes. Another feature highly appreciated by practitioners in fluid dynamics is that the discretization can be designed so that physical quantities such as momentum or mass are locally conserved. Flexibility, however, comes at a price. In particular, the memory requirements as well as the increased computational cost have discouraged wide adoption of these methods up to now. In this work we present an effective strategy to overcome these limitations inspired by classical projection methods.

Discontinuous Galerkin space discretizations of the Incompressible NavierStokes (INS) equations have been considered in several works. A mixed-order scheme on simplicial meshes has been considered by Girault, Rivière and Wheeler [21], where the authors prove LBB stability for polynomial orders up to three. More general meshes and equal-order approximation can be dealt with by suitable pressure stabilization techniques. We refer, in particular, to Cockburn Kanschat, Schötzau and Schwab [9] and to Bassi, Crivellini, Di Pietro and Rebay [4, 5]; see also [12]. Several techniques have also been proposed for the discretization of the non-linear convective term. Convergence estimates for a trilinear form with upwind stabilization have been derived by Girault, Rivière and Wheeler [21]. In [9], Cockburn, Kanschat and Schötzau prove the convergence of a fixed point iteration based on the LDG method for the Oseen equations introduced in [8] to the solution of the INS problem. More recently, Di Pietro and Ern in [13] have proposed a set of sufficient conditions on the trilinear form ensuring convergence to minimal regularity solution.

As regards time marching schemes, splitting methods have already been considered also in conjunction with (fully or partially) discontinuous space discretizations. The original pressure-correction method is due to Chorin [7] and Temam [35]; an incremental form was later proposed by Goda [22], while a second-order incremental scheme is due to Van Kan [37]. In [31, Liu and Shu propose a method for the two-dimensional INS equations in the vorticity stream-function formulation with discontinuous velocity and continuous pressure. In [33], Shahbazi, Fischer and Ethier introduce an effective threestep algebraic splitting $d G$ discretization of the INS equations in primitive variables with explicit treatment of the non-linear term in the convection diffusion step. In the cited work, it is claimed that the pressure solver related to the $\mathrm{dG}$ discretization of the projection step is the most expensive stage, and efficient preconditioning has to be devised. As this assertion is confirmed by our numerical experiments, we have opted for a continuous approximation of 
the pressure.

The focus of the work will be on moderate-to-high Reynolds flows. As noted by Karniadakis and Sherwin [27], in high Reynolds incompressible flows, splitting methods can be computationally efficient and competitive in accuracy compared to more expensive coupled methods. As a matter of fact, monolithic approaches involve the solution of the saddle point problem induced by the incompressibility constraint, which in turn requires ad-hoc preconditioners and has limited applicability to 3D large-scale unsteady simulations. Stability at high-Reynolds numbers is achieved here using a discontinuous approximation for the velocity. Discontinuous approximations are known to exhibit a better behavior when convection becomes dominant, as the additional freedom to jump across interfaces can often compensate poor grid resolution. To reduce the amount of artificial diffusion, the convective term is discretized following Di Pietro and Ern [13], where a non-dissipative formulation based on Temam's device is proposed. Interestingly enough, this choice proves robust even at considerably high Reynolds numbers. Moreover, when coupled with a continuous pressure approximation, it yields inherently inf-sup stable space couples. This renders pressure stabilization unnecessary, thereby reducing the coupling between the equations of momentum and mass conservation.

The particular form of the pressure-correction scheme proposed is inspired by Guermond and Quartapelle [24], with projection step expressed as a Poisson problem. The main difference lays in the implicit treatment of the momentum equation based on either the backward Euler method or on a second-order backward differentiation formula (in convection-dominated flows time step restrictions due to explicit or semi-implicit treatment of the convective term are too severe compared to the loss of accuracy induced by the splitting error). Both steps of the scheme can be solved with iterative methods employing standard preconditioners, resulting in an effective solution process.

\section{Solution strategy}

In this section we discuss the solution strategy in some detail. The material is organized as follows: \$2.1 contains a general overview of projection methods, $\$ 2.2$ deals with the time discretization and $\$ 2.3$ with the space discretization.

\subsection{Projection Methods}

Let $\Omega \subset \mathbb{R}^{d}, d \in\{2,3\}$, denote a bounded, connected open set, and let $t_{F}>0$ denote the final simulation time. We consider the unsteady INS 
equations with homogeneous Dirichlet boundary conditions,

$$
\begin{array}{rr}
\partial_{t} \mathbf{u}+u_{j} \partial_{j} \mathbf{u}-\nu \triangle \mathbf{u}+\nabla p=\mathbf{f} & \text { in } \Omega \times\left(0, t_{F}\right), \\
\nabla \cdot \mathbf{u}=0 & \text { in } \Omega \times\left(0, t_{F}\right), \\
\mathbf{u}=0 & \text { on } \partial \times \times\left(0, t_{F}\right), \\
\mathbf{u}(\cdot, t=0)=\mathbf{u}_{0}, & \text { in } \Omega, \\
\langle p\rangle_{\Omega}=0, &
\end{array}
$$

where $\nu>0$ denotes the (constant) viscosity, $\mathbf{f}$ is a given body force, $u_{0}$ is the initial condition, and $\langle\cdot\rangle_{\Omega}$ denotes the average value over $\Omega$. In (1) and throughout the paper we shall use Einstein's notation to understand summations on repeated indices. The main idea of projection methods is to decouple the solution of the momentum equation (1a) from the enforcement of the incompressibility constraint (1b). Such a strategy is attractive because it only requires to solve a convection-diffusion equation for the velocity and an elliptic equation for the pressure at each time step, thereby circumventing the need for a monolithic solver. The decoupling is achieved by introducing a projection operator onto the space of divergence-free functions,

$$
D \stackrel{\text { def }}{=}\left\{\mathbf{v} \in\left[L^{2}(\Omega)\right]^{d} \mid \boldsymbol{\nabla} \cdot \mathbf{v}=0 \text { in } \Omega, \mathbf{v} \cdot \mathbf{n}=0 \text { on } \partial \Omega\right\},
$$

and using the classical decomposition

$$
\left[L^{2}(\Omega)\right]^{d}=D \oplus \nabla\left(H^{1}(\Omega)\right) .
$$

Equation (2) states that every function $\mathbf{v} \in\left[L^{2}(\Omega)\right]^{d}$ can be uniquely decomposed into the direct sum of a divergence-free component plus an irrotational one. Denote by $\mathcal{P}_{D}:\left[L^{2}(\Omega)\right]^{d} \rightarrow D$ the operator that maps every function of $\left[L^{2}(\Omega)\right]^{d}$ into its divergence free part. For a given $\mathbf{v} \in\left[L^{2}(\Omega)\right]^{d}$, this projection can be computed by solving the following Neumann problem for the potential $\phi \in H^{1}(\Omega)$ :

$$
\begin{aligned}
\triangle \phi & =\boldsymbol{\nabla} \cdot \mathbf{v} & & \text { in } \Omega, \\
\partial_{\mathbf{n}} \phi & =\mathbf{v} \cdot \mathbf{n} & & \text { on } \partial \Omega, \\
\langle\phi\rangle_{\Omega} & =0, & &
\end{aligned}
$$

and setting

$$
\mathcal{P}_{D}(\mathbf{v})=\mathbf{v}-\nabla \phi .
$$

Applying the divergence operator to the momentum equation (1a) and using the divergence-free constraint $(1 \mathrm{~b})$, we formally obtain the Poisson equation for the pressure

$$
\triangle p=\nabla \cdot\left(\mathbf{f}-u_{j} \partial_{j} \mathbf{u}\right) \quad \text { in } \Omega \times\left(0, t_{F}\right),
$$


while taking the normal component on $\partial \Omega$ and using the homogeneous Dirichlet boundary conditions yields

$$
\partial_{\mathbf{n}} p=(\mathbf{f}+\nu \triangle \mathbf{u}) \cdot \mathbf{n} \quad \text { on } \partial \Omega \times\left(0, t_{F}\right) .
$$

In writing the above expression we have assumed that the normal derivative of the pressure, the forcing term and the Laplacian of the velocity are defined almost everywhere on $\partial \Omega$. Whenever this is not the case, equation $4 \mathrm{~b}$ ) has to be intended in the sense of the duality pairing between $H^{-\frac{1}{2}}(\partial \Omega)$ and $H^{\frac{1}{2}}(\partial \Omega)$. Note, however, that Cattabriga's regularity for the exact solution [6] is needed to estimate the convergence rate. In the context of projection methods, a modified form of the problem (4) is verified by the pressure approximation, as discussed in the following section.

\subsection{Pressure-correction scheme and time integration}

This section addresses the time discretization by the pressure correction method and pinpoints the relation between the semi-discrete formulation and the computation of $\mathcal{P}_{D}$. More precisely, for brevity of exposition, we first discuss the incremental form of the pressure-correction with backward Euler time discretization, and then propose a possible generalization relying on a second-order backward differentiation formula (BDF). We introduce a partition of the time domain $\left(0, t_{F}\right)$ into equally spaced intervals of length $\Delta t$ and set, for $n>0, t^{n} \stackrel{\text { def }}{=} n \Delta t$. Let $\left(\widetilde{\mathbf{u}}^{0}, \mathbf{u}^{0}, p^{0}\right)$ denote a set of sufficiently accurate initial guesses matching similar assumptions as the ones in [24, eqs. (5.8) and (5.18)] with $H^{1}$-norm replaced by the $\|\cdot\|_{\mathrm{dG}}$-norm defined by (14).

We define the sequence of triplets $\left(\widetilde{\mathbf{u}}^{n+1}, \mathbf{u}^{n+1}, p^{n+1}\right)$ iteratively by solving the following problems:

$$
\begin{array}{rlrl}
\frac{\mathbf{u}^{n+1}-\widetilde{\mathbf{u}}^{n}}{\Delta t}-\nu \Delta \mathbf{u}^{n+1}+\left(u_{j}^{n+1} \partial_{j}\right) \mathbf{u}^{n+1}+\frac{1}{2}\left(\boldsymbol{\nabla} \cdot \mathbf{u}^{n+1}\right) \mathbf{u}^{n+1}+\nabla p^{n} & =\mathbf{f}^{n+1} & & \text { in } \Omega, \\
\mathbf{u}^{n+1} & =0 & \text { on } \partial \Omega,
\end{array}
$$

and

$$
\begin{aligned}
\frac{\widetilde{\mathbf{u}}^{n+1}-\mathbf{u}^{n+1}}{\Delta t}+\nabla\left(p^{n+1}-p^{n}\right) & =0 & & \text { in } \Omega, \\
\nabla \cdot \widetilde{\mathbf{u}}^{n+1} & =0 & & \text { in } \Omega, \\
\widetilde{\mathbf{u}}^{n+1} \cdot \mathbf{n} & =0 & & \text { on } \partial \Omega .
\end{aligned}
$$


In (5a), Temam's device [35] has been used to obtain a skew-symmetric version of the convective term. In practice, the projection step (6) is performed by solving a Poisson problem on the pressure increment supplemented by homogeneous boundary conditions, as detailed in what follows. Both $\widetilde{\mathbf{u}}^{n}$ and $\mathbf{u}^{n}$ represent approximations of the exact velocity at the discrete time $t^{n}$. In particular, $\mathbf{u}^{n}$ accounts for momentum diffusion and convection as well as for the exact boundary condition, whereas $\widetilde{\mathbf{u}}^{n}$ incorporates the divergence-free constraint. It can be checked that the projection step $(6)$ is equivalent to setting $\widetilde{\mathbf{u}}^{n+1}=\mathcal{P}_{D} \mathbf{u}^{n+1}$, thereby showing that the algorithm (5)-(6) belongs to the class of projection methods.

Following Guermond and Quartapelle [23], our implementation does not require the actual computation of $\widetilde{\mathbf{u}}^{n}$. As a matter of fact, from equation (6a) it is inferred that, for $n \geq 1$,

$$
\widetilde{\mathbf{u}}^{n}=\mathbf{u}^{n}-\Delta t \nabla\left(p^{n}-p^{n-1}\right) .
$$

Plugging the above expression for $\widetilde{\mathbf{u}}^{n}$ into $5 \mathrm{a}$ ) yields

$$
\begin{aligned}
\frac{\mathbf{u}^{n+1}-\mathbf{u}^{n}}{\Delta t}-\nu \Delta \mathbf{u}^{n+1}+\left(u_{j}^{n+1} \partial_{j}\right) \mathbf{u}^{n+1} & & \\
+\frac{1}{2}\left(\nabla \cdot \mathbf{u}^{n+1}\right) \mathbf{u}^{n+1} & =\mathbf{f}^{n+1}-\nabla\left(2 p^{n}-p^{n-1}\right) & \text { in } \Omega, \\
\mathbf{u}^{n+1} & =0 & \text { on } \partial \Omega .
\end{aligned}
$$

Applying the divergence operator to (6a), the projection step can be reformulated as a Poisson equation for the pressure increment $\left(p^{n+1}-p^{n}\right)$,

$$
\begin{aligned}
-\triangle\left(p^{n+1}-p^{n}\right) & =-\frac{1}{\Delta t} \boldsymbol{\nabla} \cdot \mathbf{u}^{n+1} & & \text { in } \Omega, \\
\partial_{\mathbf{n}}\left(p^{n+1}-p^{n}\right) & =0 & & \text { on } \partial \Omega, \\
\left\langle p^{n+1}-p^{n}\right\rangle_{\Omega} & =0 . & &
\end{aligned}
$$

The homogeneous Neumann boundary condition (8b) is obtained taking the inner product of (6a) by the outward normal $\mathbf{n}$ and using (6c) to conclude, whereas condition (8c) descends from (1e). The pressure approximation $p^{n+1}$ can thus be obtained as a result of the projection process avoiding the direct discretization of problem (4). However, it is inferred from (8b) that

$$
\nabla p^{n+1} \cdot \mathbf{n}=\nabla p^{n} \cdot \mathbf{n}=\ldots=\nabla p^{0} \cdot \mathbf{n} \quad \text { on } \partial \Omega .
$$

This boundary condition is clearly different from the consistent one derived in (4b), and it is responsible for the appearance of a spurious boundary layer in the approximate solution (cf. Orszag [32]). It has been demonstrated by 
Shen [34] and E and Liu [14] that enforcing an artificial boundary condition in a projection method limits the temporal accuracy of the pressure to first order, whereas second order can still be attained for the velocity. In the context of finite element methods, the enforcement of the consistent boundary condition requires either to resort to more expensive projection methods [25] or to adopt alternative formulations [15]. Interestingly enough, however, due to the presence of viscosity in the right hand side of equation $(4 \mathrm{~b})$, the accuracy loss ascribed to the imposition of the artificial boundary condition (9) decreases with the increase in the Reynolds number. Indeed, that $\partial_{\mathbf{n}} p=0$ on solid, stationary no-slip walls (with no body force) is a good approximation at large Re is one of the cornerstones of boundary layer theory and one of the major results of the Blasius solution on a flat plate (widely validated with experimental results). This aspect limits the benefits provided by costly projection formulations when dealing with convection-dominated flows and will be numerically investigated in $\$ 3.2$.

An alternative form of the pressure-correction algorithm can be obtained replacing the backward Euler scheme by a BDF2 formula, thereby modifying (7) as follows:

$$
\begin{gathered}
\left(\frac{\beta_{0}}{\Delta t}-\nu \triangle\right) \mathbf{u}^{n+1}+\left(u_{j}^{n+1} \partial_{j}\right) \mathbf{u}^{n+1}+\frac{1}{2}\left(\nabla \cdot \mathbf{u}^{n+1}\right) \mathbf{u}^{n+1}= \\
\mathbf{f}^{n+1}-\left(\frac{\beta_{1}}{\Delta t} \mathbf{u}^{n}+\frac{\beta_{2}}{\Delta t} \mathbf{u}^{n-1}\right)-\nabla\left(\gamma_{1} p^{n}+\gamma_{2} p^{n-1}+\gamma_{3} p^{n-2}\right) \\
\mathbf{u}^{n+1}=0 \quad \text { in } \Omega
\end{gathered}
$$

where $\beta_{0}=3 / 2, \beta_{1}=-2, \beta_{2}=1 / 2$, while taking $\gamma_{1}=7 / 3, \gamma_{2}=-5 / 3, \gamma_{3}=1 / 3$ yields a pressure extrapolation of suitable order (cf., e.g., Guermond, Minev and Shen [25]). To avoid time step restrictions and extrapolations for the non-linear term, problem (10) can be solved by means of the Newton method requiring the computation of the analytic Jacobian at every iteration. The projection step associated to 10 reads

$$
\begin{aligned}
-\triangle\left(p^{n+1}-p^{n}\right) & =-\frac{\beta_{0}}{\Delta t} \boldsymbol{\nabla} \cdot \mathbf{u}^{n+1} & & \text { in } \Omega, \\
\partial_{\mathbf{n}}\left(p^{n+1}-p^{n}\right) & =0 & & \text { on } \partial \Omega .
\end{aligned}
$$

All the numerical experiments of $\S 3$ are run using this second form of the method.

\subsection{Space discretization}

The fully discrete problem is based on a discontinuous Galerkin discretization of (7) combined with a standard continuous Galerkin discretization 
of (8). This choice guarantees LBB stability and it allows to benefit from the favorable properties of $\mathrm{dG}$ methods in convection-dominated regimes.

Let $\{\mathcal{T}\}_{h>0}$ denote a shape- and contact-regular, family of matching simplicial or quadrilateral/hexahedral meshes of the domain $\Omega$. The mesh faces are collected in the set $\mathcal{F}_{h}$ partitioned into $\mathcal{F}_{h}^{i} \cup \mathcal{F}_{h}^{b}$, where $\mathcal{F}_{h}^{b}$ collects the faces located on the boundary of $\Omega$, whereas, for all $F \in \mathcal{F}_{h}^{i}$ there exist $T_{1}, T_{2} \in \mathcal{T}_{h}, T_{1} \neq T_{2}$, such that $F=\partial T_{1} \cap \partial T_{2}$. For any function $\phi$ such that a (possibly two-valued) trace is defined a.e. on $F \in \mathcal{F}_{h}^{i}$, we let

$$
\llbracket \phi \rrbracket \stackrel{\text { def }}{=} \phi_{\mid T_{1}}-\phi_{\mid T_{2}}, \quad\{\phi\} \stackrel{\text { def }}{=} \frac{1}{2}\left(\phi_{\mid T_{1}}+\phi_{\mid T_{2}}\right) .
$$

On boundary faces, the jump and average operators are conventionally defined by $\llbracket \phi \rrbracket=\{\phi\}=\phi$. Finally, for $F \in \mathcal{F}_{h}^{b}, \mathbf{n}_{F}$ represents the unit outward normal to $\Omega$, whereas for $F \in \mathcal{F}_{h}^{i}, \mathbf{n}_{F}$ is defined as the unit normal pointing from $T_{1}$ to $T_{2}$ (the order of the elements sharing $F$ is arbitrary but fixed).

When $\mathcal{T}_{h}$ is simplicial, for a given polynomial degree $k \geq 1$, we define

$$
\begin{aligned}
& \mathrm{dG}(k) \stackrel{\text { def }}{=}\left\{v_{h} \in L^{2}(\Omega) \mid \forall T \in \mathcal{T}_{h}, v_{h \mid T} \in \mathbb{P}_{d}^{k}(T)\right\}, \\
& \mathrm{cG}(k) \stackrel{\text { def }}{=}\left\{q_{h} \in C^{0}(\bar{\Omega}) \mid \forall T \in \mathcal{T}_{h}, q_{h \mid T} \in \mathbb{P}_{d}^{k}(T)\right\},
\end{aligned}
$$

where $\mathbb{P}_{d}^{k}(T)$ denotes the restriction to the element $T$ of the polynomials of $d$ variables and total degree smaller or equal than $k$. The space $\mathbb{Q}_{d}^{k}(T)$ replaces $\mathbb{P}_{d}^{k}$ in the definition of $\mathrm{cG}(k)$ whenever matching quadrilateral/hexahedral meshes are used for the pressure equation.

The discrete velocity and pressure are sought, respectively, in

$$
U_{h} \stackrel{\text { def }}{=}[\mathrm{dG}(k)]^{d}, \quad P_{h} \stackrel{\text { def }}{=} \mathrm{cG}(k) / \mathbb{R} .
$$

Observe that non-matching meshes could be allowed for the (discontinuous) velocity approximation provided one disposes of an efficient projection operator onto the conforming pressure mesh. For brevity of notation, equal-order and mixed-order velocity-pressure discretizations are hereafter identified by $\mathrm{d} \mathrm{G}(k)-\mathrm{cG}(k)$ and $\mathrm{dG}(k)-\mathrm{cG}(k-1)$ respectively.

Diffusive term in the momentum equation. The diffusive term is discretized following Arnold [2], and the $k$-dependency of the penalty parameter is accounted for using a simplified version of the expression proposed by Georgoulis and Süli in [20]. For all $\mathbf{u}_{h}, \mathbf{v}_{h} \in U_{h}$, the corresponding bilinear form 
is then given by

$$
\begin{aligned}
a_{h}\left(\mathbf{u}_{h}, \mathbf{v}_{h}\right) \stackrel{\text { def }}{=} \int_{\Omega} \boldsymbol{\nabla}_{h} u_{h, j} \cdot \nabla_{h} v_{h, j}+\sum_{F \in \mathcal{F}_{h}} \frac{\eta k^{2}}{h_{F}} \int_{F} \llbracket \mathbf{u}_{h} \rrbracket \cdot \llbracket \mathbf{v}_{h} \rrbracket \\
\quad-\sum_{F \in \mathcal{F}_{h}} \int_{F}\left(\left\{\boldsymbol{\nabla}_{h} u_{h, j}\right\} \cdot \mathbf{n}_{F} \llbracket v_{h, j} \rrbracket+\left\{\boldsymbol{\nabla}_{h} v_{h, j}\right\} \cdot \mathbf{n}_{F} \llbracket u_{h, j} \rrbracket\right),
\end{aligned}
$$

where $\eta$ is a positive penalty parameter independent of both the mesh size $h$ and the polynomial degree $k$, and $h_{F} \stackrel{\text { def }}{=} \min _{T \in \mathcal{T}_{F}}|T|_{d} /|\partial T|_{d-1}$, with $\mathcal{T}_{F}$ denoting the set of elements to whose boundary $F$ belongs. In all the numerical experiments of $\S 3$ we set $\eta=3$.

Nonlinear convective term in the momentum equation. For the convective trilinear form, we follow Di Pietro and Ern [13], where a non-dissipative formulation relying on Temam's device is proposed. For all $\mathbf{w}_{h}, \mathbf{u}_{h}, \mathbf{v}_{h} \in U_{h}$ we set

$$
\begin{aligned}
t_{h}\left(\mathbf{w}_{h}, \mathbf{u}_{h}, \mathbf{v}_{h}\right) \stackrel{\text { def }}{=} \int_{\Omega} \mathbf{w}_{h} \cdot \nabla_{h} u_{h, j} v_{h, j}-\sum_{F \in \mathcal{F}_{h}^{i}} \int_{F} \mathbf{n}_{F} \cdot\left\{\mathbf{w}_{h}\right\} \llbracket \mathbf{u}_{h} \rrbracket \cdot\left\{\mathbf{v}_{h}\right\} \\
+\frac{1}{2} \int_{\Omega}\left(\boldsymbol{\nabla}_{h} \cdot \mathbf{w}_{h}\right)\left(\mathbf{u}_{h} \cdot \mathbf{v}_{h}\right)-\frac{1}{2} \sum_{F \in \mathcal{F}_{h}} \int_{F} \mathbf{n}_{F} \cdot \llbracket \mathbf{w}_{h} \rrbracket\left\{\mathbf{u}_{h} \cdot \mathbf{v}_{h}\right\},
\end{aligned}
$$

yielding a non-conservative $\mathrm{dG}$ method that contains a source term proportional to the divergence of the discrete velocity (still converging to zero as the mesh is refined). A conservative variant requiring the non-standard modification of the pressure field first proposed in [9] has been devised in [13, $\S 5.4]$.

Discrete velocity divergence and discrete pressure gradient. The bilinear form associated to the pressure-velocity coupling terms is defined, for all $\left(\mathbf{v}_{h}, q_{h}\right) \in U_{h} \times P_{h}$ as

$$
b_{h}\left(\mathbf{v}_{h}, q_{h}\right) \stackrel{\text { def }}{=}-\int_{\Omega} \mathbf{v}_{h} \cdot \nabla q_{h}=\int_{\Omega}\left(\nabla_{h} \cdot \mathbf{v}_{h}\right) q_{h}-\sum_{F \in \mathcal{F}_{h}} \int_{F} \mathbf{n}_{F} \cdot \llbracket \mathbf{v}_{h} \rrbracket\left\{q_{h}\right\} .
$$

Remark 1 (LBB stability). Let, for all $\mathbf{v}_{h} \in U_{h}$,

$$
\left\|\mathbf{v}_{h}\right\|_{\mathrm{dG}}^{2} \stackrel{\text { def }}{=} \sum_{i=1}^{d}\left(\left\|\nabla_{h} v_{h, i}\right\|_{L^{2}(\Omega)}^{2}+\sum_{F \in \mathcal{F}_{h}^{i}} h_{F}^{-1}\left\|\llbracket v_{h, i} \rrbracket\right\|_{L^{2}(F)}^{2}\right) .
$$


That $\|\cdot\|_{\mathrm{dG}}$ is a norm on $U_{h}$ is a classical result (see [2, Lemma 2.1]). To check the discrete inf-sup condition, fix $q_{h} \in P_{h}$. Following Nečas [26], there exists $\mathbf{v}_{q_{h}} \in\left[H_{0}^{1}(\Omega)\right]^{d}$ such that $\boldsymbol{\nabla} \cdot \mathbf{v}_{q_{h}}=q_{h}$ almost everywhere in $\Omega$ and $\left\|\mathbf{v}_{q_{h}}\right\|_{\left[H^{1}(\Omega)\right]^{d}} \leq C\left\|q_{h}\right\|_{L^{2}(\Omega)}$ with $C$ independent of the mesh size $h$. Then,

$$
\begin{aligned}
\left\|q_{h}\right\|_{L^{2}(\Omega)}^{2} & =\int_{\Omega}\left(\boldsymbol{\nabla} \cdot \mathbf{v}_{q_{h}}\right) q_{h}=-\int_{\Omega} \mathbf{v}_{q_{h}} \cdot \boldsymbol{\nabla} q_{h} \\
& =-\int_{\Omega} \pi_{h} \mathbf{v}_{q_{h}} \cdot \boldsymbol{\nabla} q_{h}=b_{h}\left(\pi_{h} \mathbf{v}_{q_{h}}, q_{h}\right) \\
& \leq \sup _{\mathbf{w}_{h} \in U_{h}} \frac{b_{h}\left(\pi_{h} \mathbf{v}_{q_{h}}, q_{h}\right)}{\left\|\mathbf{w}_{h}\right\|_{\mathrm{dG}}}\left\|\pi_{h} \mathbf{v}_{q_{h}}\right\|_{\mathrm{dG}} \leq C \sup _{\mathbf{w}_{h} \in U_{h}} \frac{b_{h}\left(\mathbf{w}_{h}, q_{h}\right)}{\left\|\mathbf{w}_{h}\right\|_{\mathrm{dG}}}\left\|q_{h}\right\|_{L^{2}(\Omega)},
\end{aligned}
$$

where we have used the fact that $P_{h} \subset \mathrm{dG}(k)$ to replace $\mathbf{v}_{q_{h}}$ with its $L^{2}$ projection $\pi_{h} \mathbf{v}_{q_{h}}$ onto $U_{h}$ in the second line, and concluded using the $H^{1}$-stability of the $L^{2}$-projector to bound $\left\|\pi_{h} \mathbf{v}_{q_{h}}\right\|_{\mathrm{dG}}$ with $\left\|\mathbf{v}_{q_{h}}\right\|_{\left[H^{1}(\Omega)\right]^{d}}$ (and, hence, with $\left.C\left\|q_{h}\right\|_{L^{2}(\Omega)}\right)$. Observe that the above argument does not entail restrictions on the polynomial degree $k$ in $(12)$. This is so because the pressure is sought in a continuous space. When considering discontinuous pressures and triangular meshes, the space couples $\mathrm{dG}(k)-\mathrm{dG}(k-1)$ are stable only up to $k=3$ (see Girault, Rivière, and Wheeler [19]). We refer to Toselli [36] for a comprehensive study of fully discontinuous space couples on quadrilateral and hexahedral meshes. Stabilized formulations allowing equal-order, fully discontinuous approximations can be found, e.g., in [4, 5, 10, 8, 9, 12, 13.

Finally, the bilinear form associated to the time derivative discretization is

$$
m_{h}\left(\mathbf{u}_{h}, \mathbf{v}_{h}\right) \stackrel{\text { def }}{=} \int_{\Omega} \mathbf{u}_{h} \cdot \mathbf{v}_{h} .
$$

The discrete equivalent of equation 10 consist in seeking $\mathbf{u}_{h}^{n+1} \in U_{h}$ such that

$$
\begin{gathered}
\frac{\beta_{0}}{\Delta t} m_{h}\left(\mathbf{u}_{h}^{n+1}, \mathbf{v}_{h}\right)+\nu a_{h}\left(\mathbf{u}_{h}^{n+1}, \mathbf{v}_{h}\right)+t_{h}\left(\mathbf{u}_{h}^{n}, \mathbf{u}_{h}^{n+1}, \mathbf{v}_{h}\right)+t_{h}\left(\mathbf{u}_{h}^{n+1}, \mathbf{u}_{h}^{n}, \mathbf{v}_{h}\right)= \\
\int_{\Omega} \mathbf{f} \mathbf{v}_{h}-\frac{1}{\Delta t} m\left(\mathbf{u}_{h}^{*}, \mathbf{v}_{h}\right)+b_{h}\left(\mathbf{v}_{h}, p_{h}^{*}\right)+t_{h}\left(\mathbf{u}_{h}^{n}, \mathbf{u}_{h}^{n}, \mathbf{v}_{h}\right), \quad \forall \mathbf{v}_{h} \in U_{h}, \quad
\end{gathered}
$$

where we have set $\mathbf{u}_{h}^{*} \stackrel{\text { def }}{=} \beta_{1} \mathbf{u}_{h}^{n}+\beta_{2} \mathbf{u}_{h}^{n-1}$ and $p_{h}^{*} \stackrel{\text { def }}{=} \gamma_{1} p_{h}^{n}+\gamma_{2} p_{h}^{n-1}+\gamma_{3} p_{h}^{n-2}$, with the coefficients $\beta_{i}$ and $\gamma_{i}$ defined in section 2.2. Finally, the discrete problem corresponding to equation (11) consists in seeking $p_{h}^{n+1} \in P_{h}$ such that

$$
\int_{\Omega} \boldsymbol{\nabla}_{h} p_{h}^{n+1} \cdot \nabla_{h} q_{h}=-\frac{\beta_{0}}{\Delta t} b_{h}\left(\mathbf{u}_{h}^{n+1}, q_{h}\right)+\int_{\Omega} \boldsymbol{\nabla}_{h} p_{h}^{n} \cdot \nabla_{h} q_{h}, \quad \forall q_{h} \in P_{h},
$$

with the coefficient $\beta_{0}$ defined in section 2.2 . 


\section{Numerical validation}

In order to demonstrate the effectiveness of the solution method in the context of convection-dominated incompressible flows, we now present temporal and spatial accuracy results obtained for a set of classical benchmark cases.

\subsection{Implementation}

The solver is implemented using the tools provided by the libMesh open source finite element library [29]. For the approximating polynomial space $\mathbb{P}_{d}^{k}(T)$ we use monomials for the discontinuous spaces $\mathrm{dG}(k)$ and Lagrange polynomials for the continuous spaces $\mathrm{cG}(k)$. Thus, velocity is approximated by modal shape functions, while pressure is discretized by means of nodal shape functions. The quadrature points are computed using a Gaussian quadrature formula, and the quadrature order is set so that the mass matrix is integrated exactly. The quadrature order is not increased to take into account exact integration of the non-linear term.

Parallelization is provided by the libMesh library at the assembly level, while data structures and algorithms for the parallel solution of sparse linear systems are provided by the PETSc toolkit [3]. Both levels make use of the MPI communication protocol following a distributed memory model. Mesh partitioning is performed using the METIS library [28] or its parallel counterpart ParMETIS.

\subsection{Temporal accuracy}

In order to confirm the temporal accuracy estimates derived by E and Liu [14], we consider the Taylor vortex test along with the benchmark problem proposed by Couzy [11].

Taylor vortex temporal test. The aim of this test is to demonstrate that the scheme (15)-16) allows to march in time with high CFL numbers independently of the Reynolds number, while preserving the expected temporal accuracy. The computational domain consist of the space-time cylinder $\Omega \times(0.1,6.1)$ with $\Omega=(-\pi / 2, \pi / 2)^{2}$. The Dirichlet boundary conditions as well as the initial condition are deduced from the exact solution

$$
\begin{aligned}
& \mathbf{u}=[-\cos (\pi x) \sin (\pi y) \mathbf{i}+\sin (\pi x) \cos (\pi y) \mathbf{j}] e^{-2 \pi \nu t}, \\
& p=-\cos (2 \pi x) \cos (2 \pi y) e^{-4 \pi \nu t}
\end{aligned}
$$

where $\{\mathbf{i}, \mathbf{j}\}$ denotes the canonical basis of $\mathbb{R}^{2}$. The space discretization relies on a $\mathrm{dG}(2)-\mathrm{cG}(2)$ approximation on a very fine $300 \times 300$ quadrilateral grid, yielding $L^{2}$-projection errors of the order of $10^{-8}$ for the exact solution $(\mathbf{u}, p)$. 


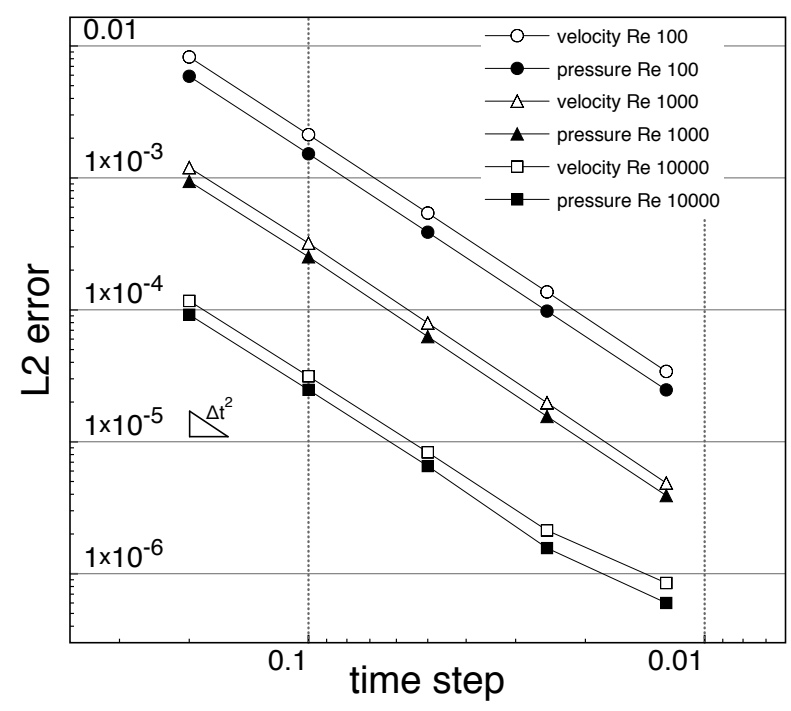

Figure 1: Temporal accuracy of the scheme evaluated using the unsteady Navier-Stokes Taylor vortex analytical solution (see text for details).

The simulations were run with time steps $\Delta t \in\{0.2,0.1,0.05,0.025,0.0125\}$ and Reynolds number $\operatorname{Re} \in\left\{10^{2}, 10^{3}, 10^{4}\right\}$. The results are summarized in Figure 1. The convergence rate for the velocity and the pressure error in $L^{2}$ norm, evaluated at the final simulation time $t_{F}=6.1$, is 2 for all but the smaller time step at $R e=10^{4}$, where the spatial error starts to dominate. The maximum CFL number, corresponding to the largest time step and computed as proposed in [27] for a convection model problem, is about 50 . The implicit treatment of the non-linear term allows to choose $\Delta t$ according to accuracy considerations related to the splitting error and to the physics to be modeled instead of dealing with the stringent stability limit of the convection-diffusion operator.

Couzy decoupling error temporal test. The second test aimed at assessing the accuracy of the proposed splitting method is the one proposed by Couzy [11]. The INS equations are solved on the time-space cylinder $\Omega \times(0,0.75)$, with space domain $\Omega=(0,1)^{2} \backslash(0.4,0.6)^{2}$ consisting of a square with a hole in the middle. We consider the exact solution

$$
\begin{aligned}
& \mathbf{u}=[-\cos (\pi x / 2) \sin (\pi y / 2) \mathbf{i}+\sin (\pi x / 2) \cos (\pi y / 2) \mathbf{j}] \sin (\pi t), \\
& p=-\pi \sin (\pi x / 2) \sin (\pi y / 2) \sin (\pi t) .
\end{aligned}
$$

The forcing term as well as the Dirichlet boundary conditions and the initial condition are deduced from the above expression. The problem is discretized in space using $\mathrm{dG}(2)-\mathrm{cG}(2)$ elements on a fine grid composed by 

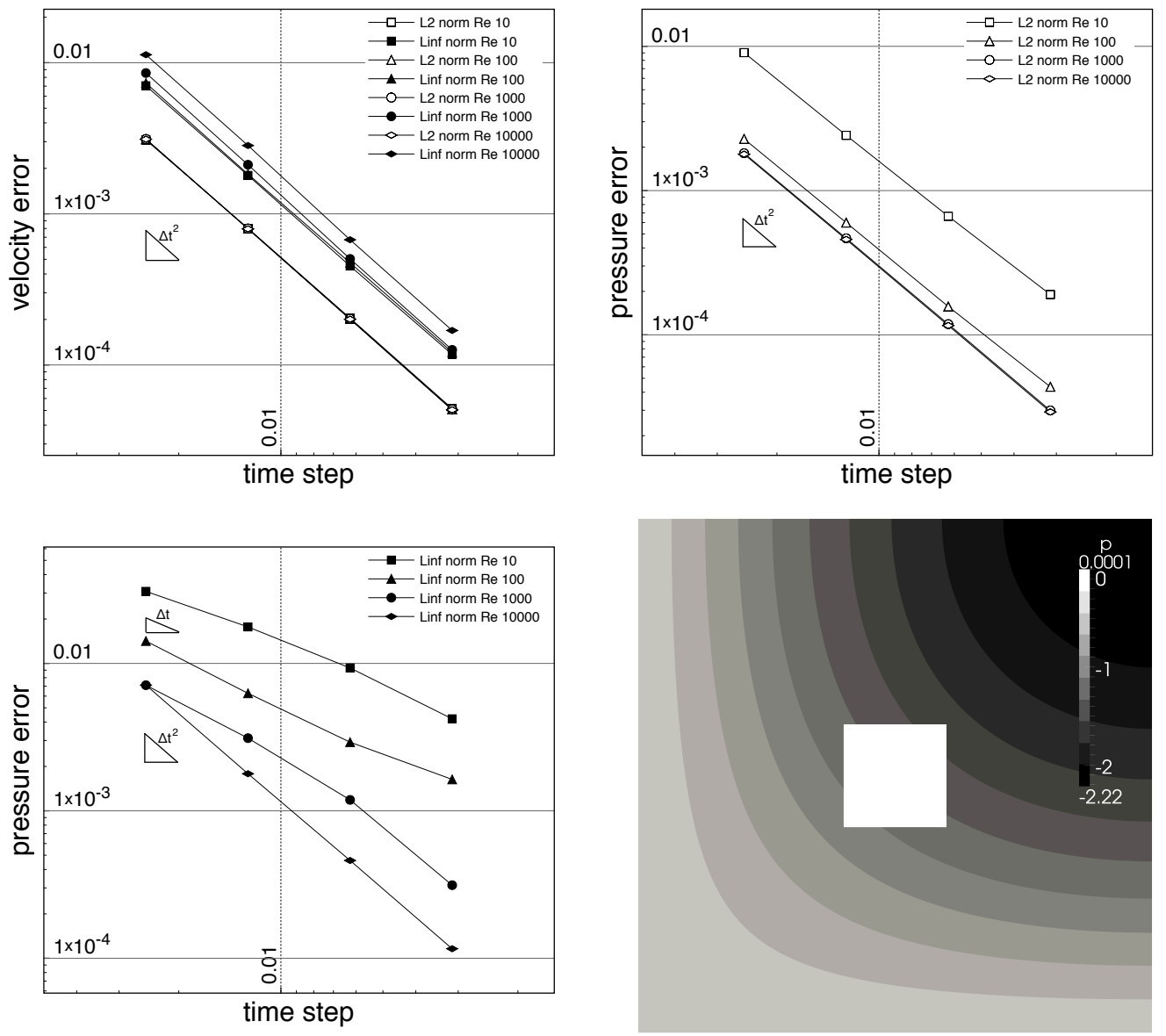

Figure 2: Couzy test case. Top row and bottom row left, temporal accuracy of the scheme evaluated on the unsteady Navier-Stokes problem proposed by Couzy (see text for details). Bottom row right, contours of the pressure field at $\mathrm{Re}=10000$, time integration performed with the smallest time step (see text for details). 
24,400 quadrilaterals, ensuring that spatial errors are dominated by temporal errors. The $L^{2}$ and $L^{\infty}$ error for both velocity and pressure are evaluated at the final simulation time $t_{F}=0.75$ using increased-order quadrature rules to provide the required accuracy. The simulation is run for Reynolds number $\operatorname{Re} \in\left\{10,10^{2}, 10^{3}, 10^{4}\right\}$, corresponding to $\nu=\left\{10^{-1}, 10^{-2}, 10^{-3}, 10^{-4}\right\}$. The results are summarized in Figure 2. The slope of the fit for the velocity in $L^{2}$ and $L^{\infty}$ norm and for the pressure in $L^{2}$ norm is almost 2 . Interestingly enough, while the velocity error is almost independent of the Reynolds number, the pressure error decreases with the increase of the Reynolds number. Moreover, at low Reynolds numbers, the convergence rate for the pressure in $L^{\infty}$ norm is somewhere in between the order of velocity and the order of velocity minus one, confirming the expected convergence rate, while at $R e=10^{4}$ it exhibits full second order. This behavior is referable to the pressure boundary layer due to the artificial Neumann boundary condition derived in equation (9). In the vanishing viscosity limit, equation (9) enforces the consistent boundary condition in equation (4b) so that the splitting error limiting the accuracy of the pressure vanishes.

\subsection{Spatial accuracy}

To numerically assess the spatial convergence rates, we consider the Kovasznay flow [30] in the two-dimensional domain $(-0.5,1.5) \times(0,2)$ and the solution proposed by Ethier and Steinman [17] in the three-dimensional domain $(-1,1)^{3}$. In these test cases we deal with low Reynolds number flows (as a matter of fact, the Kovasznay solution is similar to the low-speed flow of a viscous fluid past an array of cylinders, while the three-dimensional solution consists in a series of counter-rotating vortices involving all three Cartesian velocity components). The exact solution of the Kovasznay flow is given by

$$
\mathbf{u}=1-e^{\lambda x} \cos (2 \pi y) \mathbf{i}+\frac{\lambda}{2 \pi} e^{\lambda x} \sin (2 \pi y) \mathbf{j}, \quad p=\frac{1}{2}\left(1-e^{2 \lambda x}\right),
$$



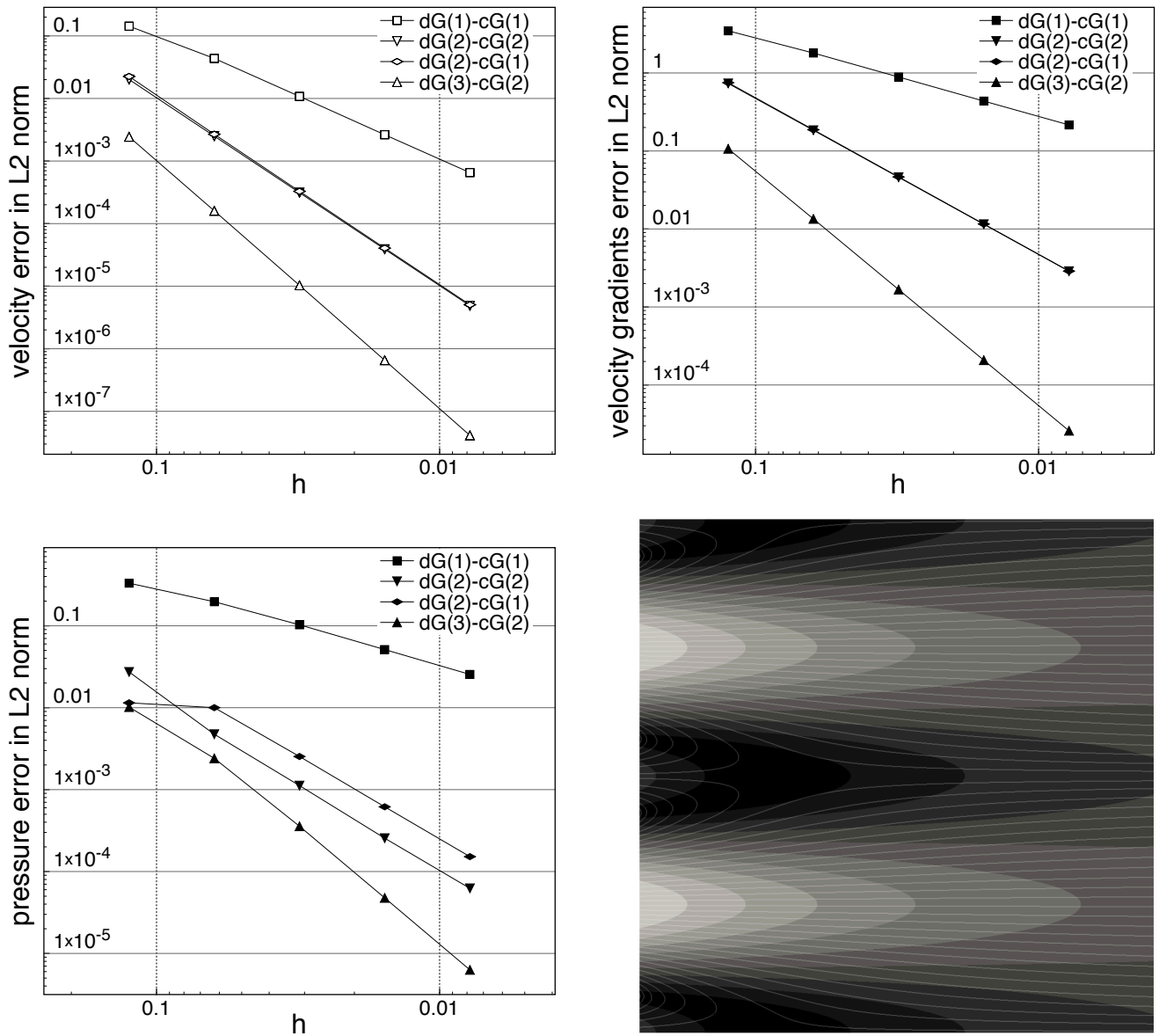

Figure 3: Kovasznay test case. Top row and bottom row left, spatial accuracy of the finite element discretization evaluated on the steady Navier-Stokes problem proposed by Kovasznay (see text for details). Bottom row right, streamlines and velocity contours. 

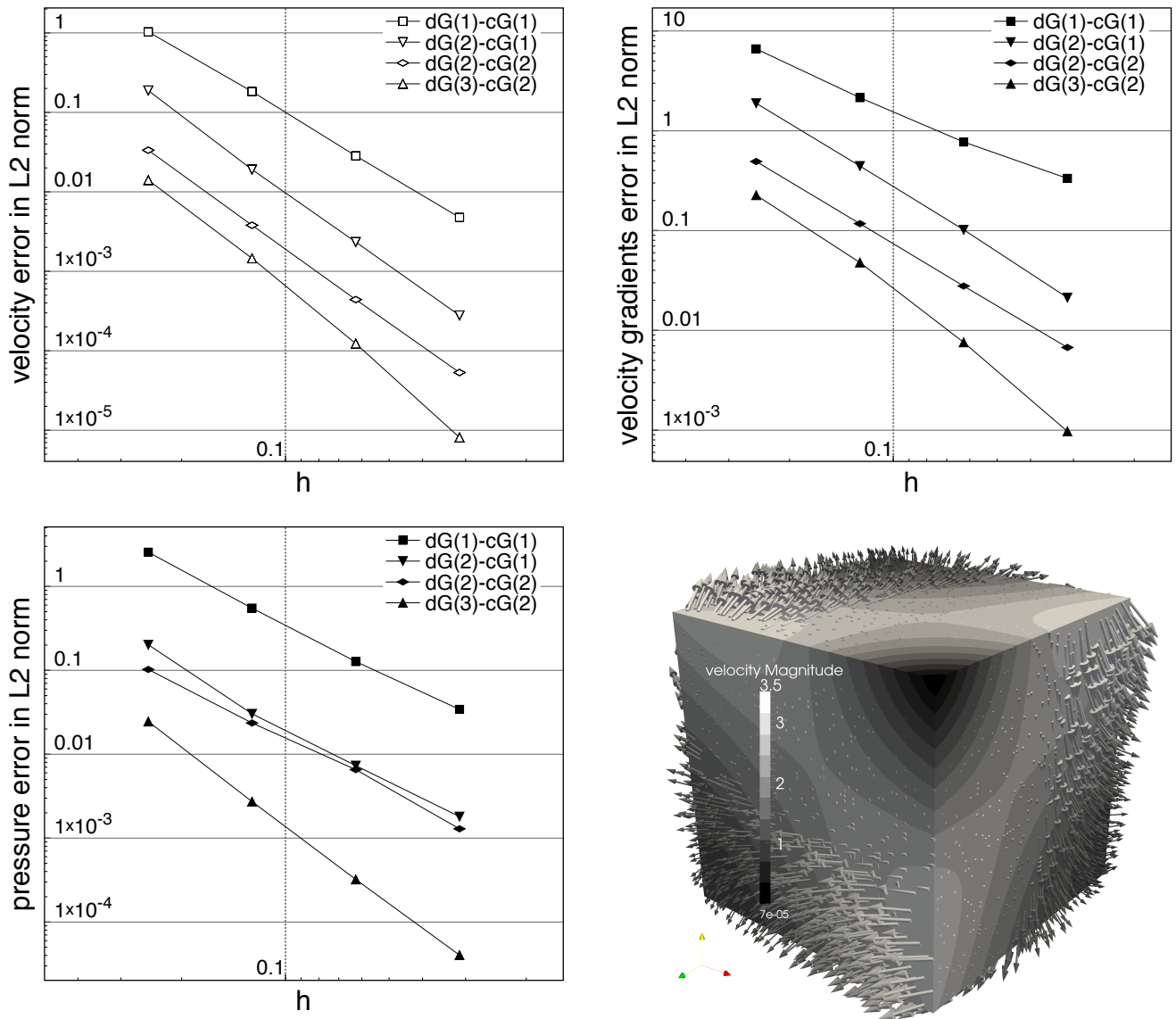

Figure 4: Ethier-Steinman test case. Top row and bottom row left, spatial accuracy of the finite element discretization evaluated on the steady Navier-Stokes problem proposed by Ethier and Steinman (see text for details). Bottom row right, velocity contours and velocity vectors. 
where $\lambda=\frac{1}{2 \nu}-\left(\frac{1}{4 \nu^{2}}+4 \pi^{2}\right)^{\frac{1}{2}}$. The unsteady analytical solution devised by Ethier and Steinman reads

$$
\begin{aligned}
\mathbf{u}= & -a\left(e^{a x} \sin (a y+d z)+e^{a z} \cos (a x+d y)\right) e^{-d^{2} \nu t} \mathbf{i} \\
& -a\left(e^{a y} \sin (a z+d x)+e^{a x} \cos (a y+d z)\right) e^{-d^{2} \nu t} \mathbf{j} \\
& -a\left(e^{a z} \sin (a x+d y)+e^{a y} \cos (a z+d x)\right) e^{-d^{2} \nu t} \mathbf{k} \\
p=-\frac{a^{2}}{2}( & e^{2 a x}+e^{2 a y}+e^{2 a z} \\
& +2 \sin (a x+d y) \cos (a z+d x) e^{a(y+z)} \\
& +2 \sin (a y+d z) \cos (a x+d y) e^{a(z+x)} \\
& \left.+2 \sin (a z+d x) \cos (a y+d z) e^{a(x+y)}\right) e^{-2 d^{2} \nu t},
\end{aligned}
$$

where $a=\frac{\pi}{4}$ and $d=\frac{\pi}{2}$. In order to evaluate the spatial convergence without dealing with temporal errors, we consider the solution at time $t=0$ and we add a forcing term balancing diffusion terms in the absence of unsteady terms (while convective terms balance the pressure gradient).

In both test cases we take $\nu=0.025$ (corresponding to $\mathrm{Re}=40$ ) and (weakly) enforce Dirichlet boundary according to the exact solution, while the initial conditions over the whole domain corresponds to zero velocity and pressure. In order to obtain a steady-state solution, a pseudo-time integration is performed employing a fixed time step $\Delta t=0.1$. The $L^{2}$ error for both velocity and pressure as well as the $L^{2}$ error for the velocity gradients are displayed in Figures 3 and 4 . The theoretical convergence rates of $h^{k+1}$ for the $L^{2}$ error on the velocity, of $h^{k}$ for the $L^{2}$ error on the pressure and for the $L^{2}$ error on the velocity gradients are confirmed for both the $\mathrm{dG}(k)-\mathrm{cG}(k)$ and $\mathrm{dG}(k)-\mathrm{cG}(k-1)$ discretizations. It is interesting to note that in the approximation of the three-dimensional solution proposed by Ethier and Steinman the $\mathrm{dG}(1)-\mathrm{cG}(1)$ discretization shows a second order convergence rate for the pressure error in $L^{2}$ norm while the theoretical convergence rates for the velocity underestimate the numerically evaluated ones by half an order.

Albeit the convergence rate is the same, $\mathrm{dG}(k)$-cG $(k)$ discretizations yield more precise pressure approximations than $\mathrm{dG}(k)-\mathrm{cG}(k-1)$ discretizations, scoring a point for equal order finite element spaces implementations. It has to be noticed, however, that the computational effort required by the iterative solution of the projection step can grow considerably in large scale simulations, see Section 3.5, which can render dG $(k)-\mathrm{cG}(k-1)$ discretizations more efficient in some configurations. Unlike the convection-diffusion step, the number of iterations required by the projection step strongly increases moving from $k=1$ to $k=2$, revealing the need of better preconditioning 

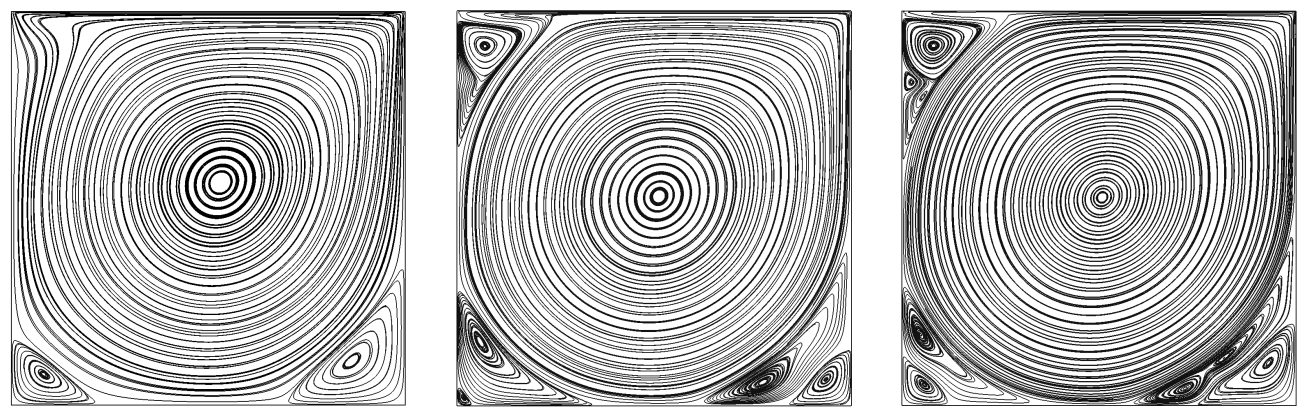

Figure 5: 2D lid-driven cavity test. Streamlines for Re 1000, 10000, 20000.
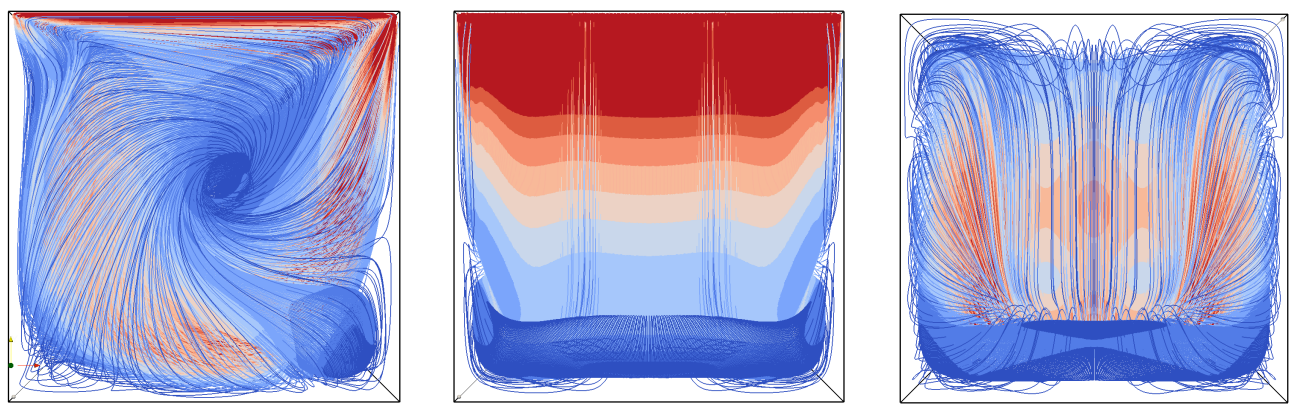

Figure 6: 3D lid-driven cavity test, Re 1000. Streamlines color-coded by velocity magnitude. Side, front and bottom view.

techniques for higher-order pressure discretizations. In serial computations it is possible to reduce the number of iterations of the GMRES iterative solver increasing the levels of fills of the ILU preconditioner, while in parallel we obtained sensible improvements relying on preconditioners based on algebraic multigrid, see e.g. [18].

\section{4. $2 D$ and $3 D$ lid-driven cavity}

As a final test case, we consider the two- and three-dimensional lid-driven cavity flow. The computational domain for this problem is a square (a cube in 3D) with edges of unit length. The top side of the cavity slides with a constant imposed velocity, while no-slip Dirichlet boundary conditions are enforced on the remaining sides. Despite its simple geometry, this lid-driven cavity flow presents complex flow patterns due to multiple recirculating secondary vortices at the corners of the cavity, see Figure 5. The space discretization is based on $\mathrm{dG}(2)-\mathrm{cG}(1)$ elements on a $120 \times 120$ quadrilateral grid in $2 \mathrm{D}$ and a $50 \times 50 \times 50$ hexahedral grid in $3 \mathrm{D}$. The simulation is advanced in 

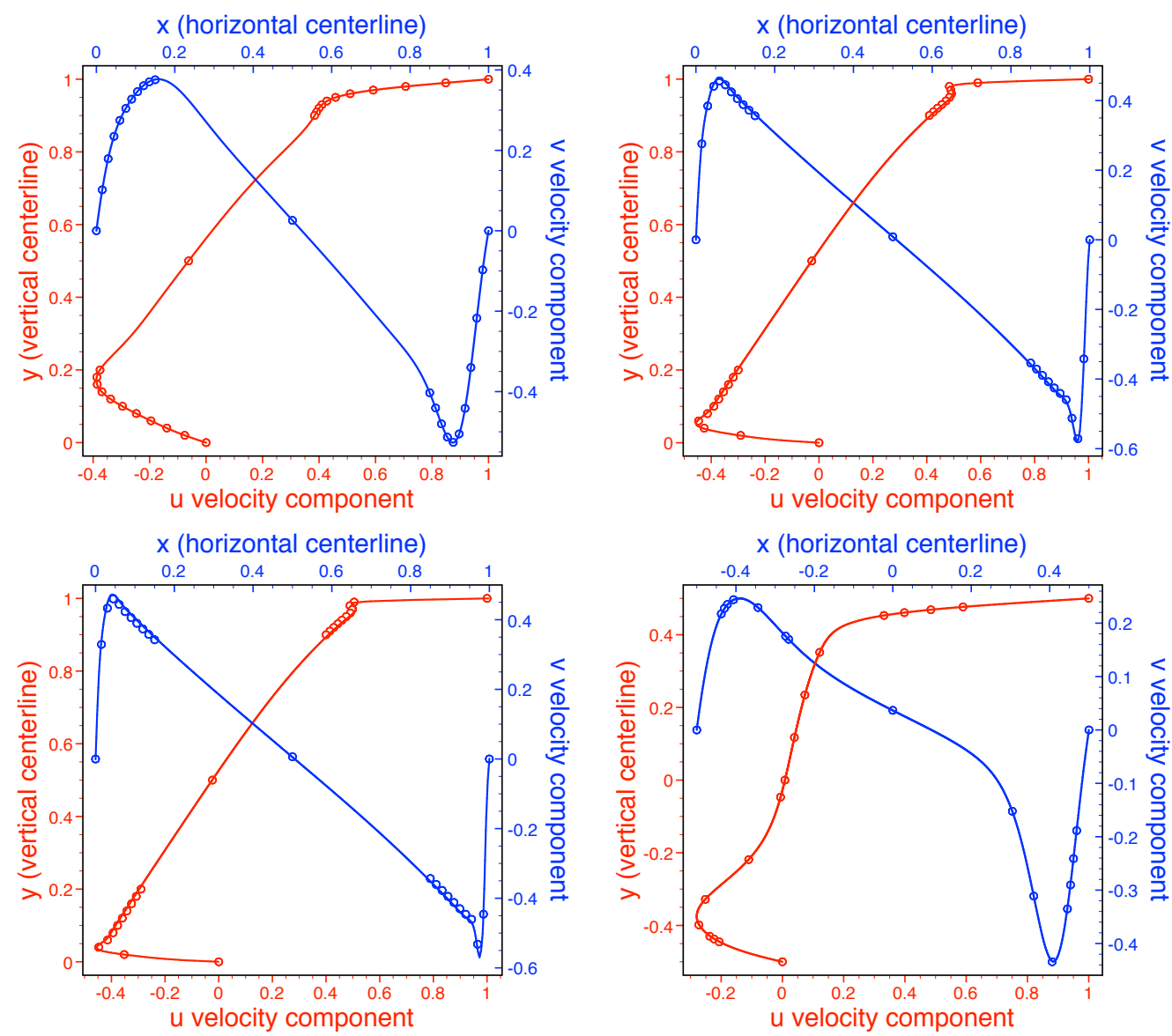

Figure 7: Lid-driven cavity test. Plots of horizontal (red) and vertical (blue) velocity components along the vertical and horizontal centerlines respectively. Top row and bottom row left, 2D lid-driven cavity at Re 1000, 10000 and 20000: the dots correspond to the reference values provided by Erturk et al [16]. Bottom row right, 3D lid-driven cavity at Re1000: the dots correspond to the reference values provided by Albensoeder et al [1]. 
time adopting a pseudo-time integration with fixed time step $\Delta t=0.1$ until a steady-state is reached.

We present 2D lid-driven cavity solutions at Reynolds number $R e=1000$, $\operatorname{Re}=10000$, and $\operatorname{Re}=20000$ and compare them with the accurate reference solutions presented in [16, where the values of the two components of the velocity are tabulated along the horizontal and vertical centerlines for $\operatorname{Re} \leq 21000$. These reference solutions have been obtained on a very fine uniform grid of $601 \times 601$ with a streamfunction and vorticity formulation of the Navier-Stokes equations. In Figure 7 it can be appreciated that velocity profiles match the reference solutions for all the Reynolds numbers here considered. As the Reynolds number increases, the gradient of the velocity at the boundary layers becomes stronger, and the number of recirculating vortices at the corners on the cavity increases. In these cases, the accuracy of the solution critically depends on modeling the shear layer along the lid and the discontinuity in the boundary condition at the upper right and left corner.

The 3D lid-driven cavity solution is computed at Reynolds number $\mathrm{Re}=1000$, and compared with the velocity results tabulated along the $\mathrm{x}$-axis and $\mathrm{y}$-axis in [1], where a projection method coupled to a fifth-order accurate Chebyshev collocation discretization on a $96 \times 96 \times 64$ hexahedral mesh is used. In Figures 6 and 7 the symmetry of the streamlines and the perfect agreement between the velocity profiles can be appreciated.

Overall, these results demonstrate that high levels of accuracy can be reached for shear-driven flows at high Reynolds numbers using a projection method.

\subsection{Solver profiling}

Splitting algorithms are well suited for unsteady simulations where the choice of the time step is driven by the dynamics of the phenomena to be modelled. The aim of this section is to provide a detailed profiling of the proposed implementation as well as a comparison between $\mathrm{dG}(k)-\mathrm{cG}(k)$ and $\mathrm{dG}(k)$ - $\mathrm{dG}(k)$ strategies. We show, in particular, that the computational cost is significantly reduced in the former case as a result of the increased efficiency of the projection step (16). We consider the unsteady analytical solution of Ethier and Steinmann (17) at $\mathrm{Re}=40$ with Dirichlet boundary conditions and initial condition deduced from (17). Albeit relatively simple, this test case highlights the trends encountered in real-life situations.

We evaluate the execution times of the $\mathrm{dG}(2)-\mathrm{cG}(2)$ discretization on three hexahedral meshes containing $40^{3}, 50^{3}, 65^{3}$ elements respectively. The discretizations top at more than 8 millions of unknown for the discontinuous velocity on the finer mesh, while the size of the problem halves on the coarser 


\begin{tabular}{|l||c|c||c|c|}
\hline \multicolumn{5}{|c|}{ Temporal convergence, Ethier Steinman test case, $\mathrm{Re}=40$} \\
\hline \hline$\Delta t$ & $L^{2}$ error pressure & order & $L^{2}$ error velocity & order \\
\hline 0.1 & 0.1096 & & 0.00936 & \\
0.05 & 0.02998 & 1.87 & 0.002398 & 1.96 \\
0.025 & 0.007912 & 1.92 & 0.0006039 & 1.99 \\
0.0125 & 0.002020 & 1.97 & 0.00015159 & 1.99 \\
\hline
\end{tabular}

Table 1: Time convergence, Ethier and Steinman solution (17), Re $=40, \mathrm{dG}(2)-\mathrm{cG}(2)$ discretization, $65^{3}$ elements hexahedral grid.

meshes. The error related to the time discretization has been evaluated at the final step for a total time integration interval $T=[0,1]$. On all the grids here considered we are able to obtain second order temporal convergence for velocity and pressure in the $L^{2}$ norm as the temporal error dominate the spatial error. Table 1 shows the results for the finest grid.

All the simulations are run in parallel on 32 processes using 4 nodes of a quad-core CPUs dual-socket cluster disposing of 16GB of RAM per node. For the solution of the linear systems resulting from the finite element discretization we rely on the well know Block Jacoby preconditioned GMRES $(x / y)$ algorithm [3] where the number Krylov spaces $(x)$ and the maximum number of iterations $(y)$ are user-defined input parameters. We prescribe a relative residual tolerance of $1 \cdot 10^{-9}$ and we guarantees that the maximum number of linear iteration is not reached so that the iterative solver has converged up to the desired tolerance. The convergence of the Newton method in (15) is reached when the discrete $L^{2}$ norm of the solution increment is smaller than $1 \cdot 10^{-2}$, which is usually achieved in a couple of non-linear iterations. This value suffices to obtain second order time convergence.

In order to motivate the choice of a $\mathrm{dG}(k)$-cG $(k)$ strategy with discontinuous velocity and continuous pressure we compare the relative amount of time spent during the pressure correction step with respect to a fully discontinuous $\mathrm{dG}(k)-\mathrm{dG}(k)$ strategy; see Tables 2 and 3 . For the sake of completeness, a fully coupled strategy has also been considered; see Table 4. We stress that the time indication includes both the matrix assembly and the solution of the linear systems, which means that in the advection-diffusion step we consider two Jacobian assembly and as GMRES solution processes as Newton iterations. It is apparent from Table 2 that the number of GMRES iterations required for the numerical solution of projection step is strongly related to number of Krylov spaces selected, which indicates that the linear system associated to the projection step is the harder to solve. Moving to the finest $65^{3}$ elements grid we are obliged to increase the number of Krylov spaces up to 250 to efficiently solve the projection step. On the contrary, the 


\begin{tabular}{|c|c|c|c|c|c|c|}
\hline \multicolumn{7}{|c|}{ Solver profiling, Ethier Steinman test case, $40^{3}$ elements grid } \\
\hline$\Delta t$ & n_ksp & avg step time & avg $n \_$it & $\%$ time & avg $n_{-}$it & $\%$ time \\
\hline \multirow{2}{*}{0.1} & 100 & $135 \mathrm{~s}$ & \multirow{2}{*}{$80+50$} & 34 & 6200 & 66 \\
\hline & 150 & $66 \mathrm{~s}$ & & 78 & 700 & 22 \\
\hline \multirow{2}{*}{0.05} & 100 & $125 \mathrm{~s}$ & \multirow{2}{*}{$60+30$} & 29 & 6000 & 71 \\
\hline & 150 & $47 \mathrm{~s}$ & & 76 & 600 & 24 \\
\hline \multirow{2}{*}{0.025} & 100 & $112 \mathrm{~s}$ & \multirow{2}{*}{$40+20$} & 29 & 5200 & 71 \\
\hline & 150 & $43 \mathrm{~s}$ & & 74 & 600 & 26 \\
\hline \multirow{2}{*}{0.0125} & 100 & $112 \mathrm{~s}$ & \multirow{2}{*}{$30+10$} & 26 & 5200 & 74 \\
\hline & 150 & $40 \mathrm{~s}$ & & 72 & 600 & 28 \\
\hline
\end{tabular}

\begin{tabular}{|c|c|c|c|c|c|c|}
\hline & $\operatorname{man} t$ & t case & $j^{3}$ elem & its grid \\
\hline & & & \multicolumn{2}{|c|}{ a-d step } & \multicolumn{2}{|c|}{ p-c step } \\
\hline$\Delta t$ & n_ksp & avg step time & avg n_it & $\%$ time & avg n_it & $\%$ time \\
\hline \multirow{2}{*}{0.1} & 150 & $206 \mathrm{~s}$ & \multirow{2}{*}{$110+70$} & 49 & 4300 & 51 \\
\hline & 200 & $113 \mathrm{~s}$ & & 79 & 700 & 21 \\
\hline \multirow{2}{*}{0.05} & 150 & $193 \mathrm{~s}$ & \multirow{2}{*}{$70+40$} & 39 & 4200 & 61 \\
\hline & 200 & $100 \mathrm{~s}$ & & 76 & 600 & 24 \\
\hline \multirow{2}{*}{0.025} & 150 & $135 \mathrm{~s}$ & \multirow{2}{*}{$50+20$} & 47 & 2200 & 53 \\
\hline & 200 & $80 \mathrm{~s}$ & & 76 & 490 & 24 \\
\hline \multirow{2}{*}{0.0125} & 150 & $111 \mathrm{~s}$ & \multirow{2}{*}{$30+10$} & 48 & 1800 & 52 \\
\hline & 200 & $77 \mathrm{~s}$ & & 75 & 490 & 25 \\
\hline
\end{tabular}

Solver profiling, Ethier Steinman test case, $65^{3}$ elements grid

\begin{tabular}{|c|c|c||c|c||c|c||}
\hline \multicolumn{2}{|c||}{} & \multicolumn{2}{c||}{ a-d step } & \multicolumn{2}{c||}{ p-c step } \\
\hline \multirow{2}{*}{0.1} & n_ksp & avg step time & avg n_it & $\%$ time & avg n_it & $\%$ time \\
& 200 & $493 \mathrm{~s}$ & \multirow{2}{*}{$140+90$} & 57 & 3100 & 43 \\
& 250 & $399 \mathrm{~s}$ & & 78 & 1000 & 22 \\
\hline \multirow{2}{*}{0.05} & 200 & $390 \mathrm{~s}$ & \multirow{2}{*}{$90+50$} & 51 & 2800 & 49 \\
& 250 & $282 \mathrm{~s}$ & & 75 & 900 & 25 \\
\hline \multirow{2}{*}{0.025} & 200 & $297 \mathrm{~s}$ & \multirow{2}{*}{$60+30$} & 54 & 1800 & 45 \\
& 250 & $226 \mathrm{~s}$ & & 67 & 800 & 33 \\
\hline \multirow{2}{*}{0.0125} & 200 & $273 \mathrm{~s}$ & \multirow{2}{*}{$40+10$} & 52 & 1800 & 47 \\
& 250 & $212 \mathrm{~s}$ & & 64 & 800 & 36 \\
\hline
\end{tabular}

Table 2: Solver profiling on the unsteady Navier-Stokes problem proposed by Ethier and Steinman [17, $R e=40, \mathrm{dG}(2)-\mathrm{cG}(2)$ discretization. From left to right, time step size, number of Krylov spaces used by the GMRES solver, average time required for a single time step, average of the GMRES linear iterations and percentage of time spent for the advection-diffusion step and for the projection step, respectively. 


\begin{tabular}{|c|c|c|c|c|c|c|}
\hline \multicolumn{7}{|c|}{ dG-dG solver, Ethier Steinman test case, $50^{3}$ elements grid } \\
\hline \multirow[b]{2}{*}{$\Delta t$} & \multirow[b]{2}{*}{ n_ksp } & \multirow[b]{2}{*}{ avg step time } & \multicolumn{2}{|c|}{ a-d step } & \multicolumn{2}{|c|}{ p-c step } \\
\hline & & & avg n_it & $\%$ time & avg $n \_$it & $\%$ time \\
\hline \multirow{2}{*}{0.1} & 200 & $589 \mathrm{~s}$ & \multirow{2}{*}{$110+70$} & 16 & $>8000$ & 84 \\
\hline & 400 & $201 \mathrm{~s}$ & & 48 & 1000 & 52 \\
\hline \multirow{2}{*}{0.05} & 200 & $543 \mathrm{~s}$ & \multirow{2}{*}{$70+40$} & 13 & $>8000$ & 87 \\
\hline & 400 & $178 \mathrm{~s}$ & & 41 & 900 & 59 \\
\hline \multirow{2}{*}{0.025} & 200 & $612 \mathrm{~s}$ & \multirow{2}{*}{$50+20$} & 10 & $>8000$ & 90 \\
\hline & 400 & $176 \mathrm{~s}$ & & 37 & 900 & 63 \\
\hline \multirow{2}{*}{0.0125} & 200 & $667 \mathrm{~s}$ & \multirow{2}{*}{$30+10$} & 9 & $>8000$ & 81 \\
\hline & 400 & $183 \mathrm{~s}$ & & 30 & 1100 & 70 \\
\hline
\end{tabular}

Table 3: Profiling on the $\mathrm{dG}-\mathrm{dG}$ pressure-correction algorithm, unsteady Navier-Stokes problem proposed by Ethier and Steinman [17, $R e=40, \mathrm{dG}(2)-\mathrm{dG}(2)$ discretization. From left to right, time step size, number of Krylov spaces used by the GMRES solver, average time required for a single time step, average of the GMRES linear iterations and percentage of time spent for the advection-diffusion step and for the projection step, respectively.

number of linear iterations required by the advection-diffusion step is relatively independent of the number of Krylov spaces, and smoothly increases with the size of the problem.

The strong influence of the GMRES parameters on the execution time makes it clear that the ability to set up an efficient solution process for the projection is of primary importance in the pressure-correction algorithm. In this context a continuous pressure discretization is to be preferred to a more expensive discontinuous pressure discretization as the cost related to iterative solution of the projection step is bounded, even employing standard preconditioners.

To corroborate this assertion, we compare the $\mathrm{dG}(2)-\mathrm{cG}(2)$ strategy with a fully discontinuous $\mathrm{dG}(2)-\mathrm{dG}(2)$ implementation of the splitting algorithm and a fully discontinuous monolithic solver. The three strategies only differ for the pressure treatment, while the discretization of the convective and diffusive term presented in Section 2.3 are unchanged. In the $\mathrm{dG}(2)-\mathrm{dG}(2)$ pressure-correction algorithm the projection step is discretized by means of the bilinear form (13) modified to include the proper boundary conditions. Tables 3 and 4 contain the execution times on the $50^{3}$ grid. It is interesting to note that the iterative solution of the projection step in the $d G(2)-d G(2)$ pressure-correction is so much more expensive compared to the $\mathrm{dG}(2)-\mathrm{cG}(2)$ formulation that the GMRES algorithm fails to converge when the number of Krylov spaces is set at 200. In this configuration, even if a number of 


\begin{tabular}{|c|c|c|c|}
\hline \multicolumn{3}{|c|}{ dG solver, Ethier Steinman test case, $50^{3}$ elements grid } \\
\hline$\Delta t$ & $\mathrm{n}_{\mathrm{kspp}}$ & avg step time & avg $\mathrm{n}$ _it \\
\hline \multirow{2}{*}{0.1} & 200 & $560 \mathrm{~s}$ & $480+370$ \\
& 400 & $587 \mathrm{~s}$ & $380+290$ \\
\hline \multirow{2}{*}{0.05} & 200 & $480 \mathrm{~s}$ & $420+330$ \\
& 400 & $461 \mathrm{~s}$ & $310+230$ \\
\hline \multirow{2}{*}{0.025} & 200 & $380 \mathrm{~s}$ & $390+120$ \\
& 400 & $340 \mathrm{~s}$ & $280+120$ \\
\hline \multirow{2}{*}{0.0125} & 200 & $335 \mathrm{~s}$ & $370+80$ \\
& 400 & $324 \mathrm{~s}$ & $280+80$ \\
\hline
\end{tabular}

Table 4: Profiling on the dG monolithic incompressible Navier-Stokes solver proposed by [13], unsteady Navier-Stokes problem proposed by Ethier and Steinman [17], $R e=40$, second order discretization. From left to right, time step size, number of Krylov spaces used by the GMRES solver, average time required for a single time step, average of the GMRES linear iterations.

maximum iteration greater than 8000 is employed, the algorithm is unable to reach the prescribed convergence rate $\left(1 \cdot 10^{-9}\right)$, thereby spoiling the pressure convergence rates at the smaller time steps. Moreover, even when the number of Krylov spaces is set at 400 the GMRES convergence is suboptimal compared to the $\mathrm{dG}(2)-\mathrm{cG}(2)$ algorithm and better preconditioning techniques would be required for the projection step. Remarkably enough, the monolithic $\mathrm{dG}$ solver outperforms the $\mathrm{dG}(2)-\mathrm{dG}(2)$ implementation when the number of Krylov spaces is set at 200. On the other hand, the dG(2)$\mathrm{cG}(2)$ strategy is almost four time faster than the monolithic solver. For the latter, an increase in the number of Krylov spaces has a reduced, possibly negative, impact on the performance as the minor number of iteration is balanced by an higher cost per iteration; see Table 4 .

\section{Conclusions}

We have presented an efficient and robust INS solver for high-Reynolds unsteady flows. Efficiency is ensured by the use of a projection method to decouple the momentum conservation equation from the incompressibility constraint, thereby allowing an effective solution process based on standard preconditioned iterative solvers. Robustness regards to the Reynolds number is guaranteed by the fully implicit dG discretization of the convectiondiffusion step.

We demonstrated the ability to accurately resolve 2D and 3D challenging benchmark problems in moderate to high Reynolds numbers flow regimes with time steps larger than the ones imposed by the CFL stability limit. 
The possibility to manage hybrid grids can be exploited to extend the solver applicability to complex geometries as the ones required for hemodynamic simulations. An open-source hemodynamics solver based on the proposed algorithm will be the subject of future work.

\section{Acknowledgements}

The authors are thankful to Luca Antiga (Mario Negri Institute for Pharmacological Research) for fruitful discussions. The research leading to these results has received funding from the European Community's Seventh Framework Programme (FP7/2007-2013:ARCH, Project n.224390).

[1] S. Albensoeder, H. C. Kuhlmann, Accurate three-dimensional lid-driven cavity flow, J. Comp. Phys. 206 (2005) 536-558.

[2] D. N. Arnold, An Interior Penalty Finite Element Method with Discontinuous Elements, SIAM J. Num. Anal. 19 (1982) 742-760.

[3] S. Balay, K. Buschelman, V. Eijkhout, W. Gropp, D. Kaushik, M. Knepley, L. C. McInnes, B. Smith, H. Zhang, PETSc Users Manual, Argonne National Laboratory, Mathematics and Computer Science Division, 2.3.3 edn.

[4] F. Bassi, A. Crivellini, D. A. Di Pietro, S. Rebay, An artificial compressibility flux for the discontinuous Galerkin solution of the incompressible Navier-Stokes equations, Comp. Phys. 218 (2006) 794-815.

[5] F. Bassi, A. Crivellini, D. A. Di Pietro and S. Rebay, An implicit highorder discontinuous Galerkin method for steady and unsteady incompressible flows, Comp. \& Fl. 36(10) (2007) 1529-1546

[6] L. Cattabriga, Su un problema al contorno relativo al sistema di equazioni di Stokes. Rend. Sem. Mat. Univ. Padova, 31 (1961) 308-340.

[7] A. J. Chorin, Numerical solution of the Navier-Stokes equations, Math. Comp. 22 (1968) 745-762.

[8] B. Cockburn, G. Kanschat, D. Schötzau, The local discontinuous Galerkin method for the Oseen equations, Math. Comp. 73 (2003) 569593.

[9] B. Cockburn, G. Kanschat, D. Schötzau, A locally conservative LDG method for the incompressible Navier-Stokes equations, Math. Comp. 74 (2005) 1067-1095. 
[10] B. Cockburn, G. Kanschat, D. Schötzau, and C. Schwab, Local Discontinuous Galerkin methods for the Stokes system, SIAM J. Numer. Anal. 40 (2002), 319-343.

[11] W. Couzy, Spectral element discretization of the unsteady Navier-Stokes equations and its iterative solutions o parallel computers, $\mathrm{PhD}$ Thesis, École Polytechnique Fédérale de Lausanne, 1995.

[12] D. A. Di Pietro, Analysis of a discontinuous Galerkin approximation of the Stokes problem based on an artificial compressibility flux, Int. J. Numer. Meth. Fluids 55 (2007) 793-813.

[13] D. A. Di Pietro, A. Ern, Discrete functional analysis tools for discontinuous Galerkin methods with application to the incompressible NavierStokes equations, Math. Comp. 79 (2010) 1303-1330.

[14] W. E, J.-G. Liu, Projection method I: Convergence and numerical boundary layers. SIAM J. Numer. Anal. 32 (1995) 1017-1057.

[15] W. E, J.-G. Liu, Gauge method for incompressible flows, Comm. Math. Sci. 1 (2003) 317-332.

[16] E. Erturk, T. C. Corke, C. Gökçöl, Numerical Solutions Of 2-D Steady Incompressible Driven Cavity Flow At High Reynolds Numbers, Inti. J. Num. Meth. Fluids 48 (2005) 747-774.

[17] C. R. Ethier, D. A. Steinman, Exact Fully 3D Navier-Stokes Solutions for Benchmarking, Inti. J. Num. Meth. Fluids 19 (1994) 369-375.

[18] R.D. Falgout, J.E. Jones, U.M. Yang, The Design and Implementation of hypre, a Library of Parallel High Performance Preconditioners, in: A.M. Bruaset, A. Tveito (Eds.), Numerical Solution of Partial Differential Equations on Parallel Computers, Springer-Verlag, 2006, pp. 267-294.

[19] V. Girault, B. Rivière, and M. F. Wheeler. A discontinuous Galerkin method with nonoverlapping domain decomposition for the Stokes and Navier-Stokes problems. Math. Comp., 74(249):53-84 (electronic), 2004.

[20] H. Georgoulis, E. Süli, Optimal Error Estimates for the $h p$-Version Interior Penalty Discontinuous Galerkin Finite Element Method, J. Sci. Comp. 30 (2007) 465-491.

[21] V. Girault, B. Rivire, and M. F. Wheeler, A discontinuous Galerkin method with nonoverlapping domain decomposition for the Stokes and Navier-Stokes problems, Math. Comp. 74 (2004) 53-84. 
[22] K. Goda, A multistep technique with implicit difference schemes for calculation two- or three-dimensional cavity flows, J. Comp. Phys. 30 (1979) 76-95.

[23] J.-L. Guermond, L. Quartapelle, On stability and convergence of projection methods based on pressure Poisson equation, Int. J. Num. Meth. Fluids, 26 (1998) 1039-1053.

[24] J.-L. Guermond, L. Quartapelle, On the approximation of the unsteady Navier-Stokes equations by finite element projection methods. Numer. Math. 80 (1998) 207-238.

[25] J. L. Guermond, P. Minev, J. Shen, An overview of projection methods for incompressible flows, Comp. Meth. Appl. Mech. Engrg. 195 (2006) 6011-6045.

[26] J. Nečas, Equations aux dérivées partielles, Presses de l'Université de Montréal, Montréal, Canada, 1965.

[27] G. E. Karniadakis, S. Sherwin, Spectral/hp Element Methods for Computational Fluid Dynamics, Oxford University Press, USA, 2004.

[28] G. Karypis, V. Kumar, MeTis: Unstructured Graph Partitioning and Sparse Matrix Ordering System, Version 4.0, http://www.cs.umn.edu/ metis.

[29] B. Kirk, J. W. Peterson, R. H. Stogner, G. F. Carey, libMesh: A C++ Library for Parallel Adaptive Mesh Refinement/Coarsening Simulations Boundary conditions for incompressible flows, Eng. Comput. 22 (2006) 237-254.

[30] L. I. G. Kovasznay, Laminar flow behind a two-dimensional grid, Proc. Camb. Phil. Soc. 44 (1948) 58-62.

[31] J.-G. Liu, C.-W. Shu, A High-Order Discontinuous Galerkin Method for 2D Incompressible Flows, J. Comp. Phys. 160 (2000) 577-596.

[32] S. A. Orszag, Spectral methods for problems in complex geometries, J. Comp. Phys. 37 (1980) 70-92.

[33] K. Shahbazi, P. F. Fischer, C. R. Ethier, A high-order discontinuous Galerkin method for the unsteady incompressible Navier-Stokes equations, J. Comp. Phys. 222 (2007) 391-407. 
[34] J. Shen, On error estimates of projection methods for the Navier-Stokes equations: second-order schemes, Math. Comput. 65 (1996) 1039-1065.

[35] R. Temam, Sur l'approximation de la solution des équations de NavierStokes par la méthode des pas fractionnaires, Arch. Ration. Mesh. Anal. 33 (1969) 377-385.

[36] A. Toselli. $h p$-finite element discontinuous Galerkin approximations for the Stokes problem. M3AS, 12 (2002) 1565-1616.

[37] J. Van Kan, A second-order accurate pressure-correction scheme for viscous incompressible flow, SIAM J. Sci. Stat. Comp. 7 (1986) 870-891. 